Tecnología y desarrollo: Electrónica digital

\title{
Tecnología y desarrollo: Electrónica digital
}

\section{Technology and development: Digital electronics}

\section{Tecnologia e desenvolvimento: Eletrônica digital}

\author{
Paola N. Sandoval-Vizuete I \\ pnsandoval@espe.edu.ec \\ Jenny P. Calvopiña-Osorio II \\ jpcalvopina1@espe.edu.ec \\ Pablo S. Cevallos-Viscaíno III \\ pasacevi@yahoo.com
}

Recibido: 12 de septiembre de 2017 * Corregido: 08 de octubre de $2017 *$ Aceptado: 30 de noviembre de 2017

I. Docente Investigador de la Unidad de Gestión de Tecnologías de la Universidad de las Fuerzas Armadas ESPE, Latacunga, Cotopaxi, Ecuador.

II. Docente de nivelación de la Universidad de las Fuerzas Armadas ESPE, Latacunga, Cotopaxi, Ecuador.

III. Docente de Inglés de la Universidad Técnica de Cotopaxi, Coordinador del Área de Inglés de la Universidad Técnica de Cotopaxi, Docente de inglés de la Unidad de Gestión de Tecnologías de la Universidad de las Fuerzas Armadas ESPE-L, Latacunga, Cotopaxi, Ecuador. 
Tecnología y desarrollo: Electrónica digital

\title{
Resumen
}

La electrónica, y principalmente la electrónica digital, ha cambiado la forma en que nos comunicamos, nos divertimos e interactuamos entre nosotros mismos; en pocas palabras, ha cambiado la forma en que vivimos. Gracias a la electrónica digital podemos almacenar y transportar a cualquier lugar nuestras canciones favoritas. Podemos almacenar nuestros momentos más preciados de manera instantánea en imágenes o videos de muy alta calidad utilizando dispositivos que caben en la palma de nuestra mano. La electrónica digital nos permite localizar y comunicarnos de manera inmediata con nuestros amigos y seres queridos en cualquier lugar y en cualquier momento. Quizás lo más importante es que gracias a los avances en electrónica digital podemos recopilar, almacenar e intercambiar información de manera inmediata, sin importar el lugar donde nos encontremos y en donde se haya generado esa información. El componente electrónico que ha contribuido de manera directa al desarrollo de los sistemas electrónicos digitales es el microprocesador. El primer microprocesador comercial apareció a principios de los años 70s e inicialmente fue utilizado en calculadoras. Los microprocesadores modernos pueden albergar físicamente hasta 10 núcleos (o cores) en una sola pastilla de circuito integrado, esto nos otorga un gran poder de cómputo para el procesamiento de imágenes y señales. Un ejemplo claro de cómo la electrónica digital ha revolucionado la manera en que nos comportamos y vivimos es el teléfono celular. El primer teléfono celular apareció en la década de los 70s, sus dimensiones eran tan grandes que fue bautizado con el nombre de "el ladrillo" (the brick).

Palabras clave: Electrónica; digital; microprocesadores; circuitos; computo.

\begin{abstract}
Electronics, and especially digital electronics, has changed the way we communicate, have fun and interact with each other; In short, the way we live has changed. Thanks to digital electronics we can store and transport our favorite songs to any place. We can store our most precious moments instantaneously in very high quality images or videos using devices that fit in the palm of our hand. Digital electronics allows us to locate and communicate immediately with our friends and loved ones anywhere and anytime. Perhaps the most important thing is that thanks to advances in digital electronics we can collect, store and exchange information immediately, no matter where we are and where that information has been generated. The electronic component that has contributed directly to the development of digital electronic systems is the microprocessor. The first commercial
\end{abstract}


Tecnología y desarrollo: Electrónica digital

microprocessor appeared in the early $70 \mathrm{~s}$ and was initially used in calculators. Modern microprocessors can physically hold up to 10 cores (or cores) in a single chip integrated circuit, this gives us a great computing power for the processing of images and signals. A clear example of how digital electronics has revolutionized the way we behave and live is the cell phone. The first cell phone appeared in the $70 \mathrm{~s}$, its dimensions were so large that it was baptized with the name of "the brick".

Keywords: Electronics; digital; microprocessors; circuits; calculation.

\section{Resumo}

A eletrônica, e principalmente a eletrônica digital, mudou a maneira como nos comunicamos, nos divertimos e interagimos uns com os outros; Em resumo, a forma como vivemos mudou. Graças à eletrônica digital, podemos armazenar e transportar nossas músicas favoritas para qualquer lugar. Nós podemos armazenar nossos momentos mais preciosos instantaneamente em imagens ou vídeos de muito alta qualidade usando dispositivos que se encaixam na palma da nossa mão. A eletrônica digital nos permite localizar e comunicar imediatamente com nossos amigos e entes queridos em qualquer lugar e a qualquer hora. Talvez o mais importante seja que, graças aos avanços na eletrônica digital, podemos coletar, armazenar e trocar informações imediatamente, não importa onde estivéssemos e onde essa informação tenha sido gerada. O componente eletrônico que contribuiu diretamente para o desenvolvimento de sistemas eletrônicos digitais é o microprocessador. O primeiro microprocessador comercial apareceu no início dos anos 70 e foi inicialmente usado em calculadoras. Os microprocessadores modernos podem sustentar fisicamente até 10 núcleos (ou núcleos) em um circuito integrado de chip único, o que nos dá uma excelente potência de computação para processamento de imagens e sinais. Um exemplo claro de como a eletrônica digital revolucionou a maneira como nos comportamos e vivemos é o celular. O primeiro celular apareceu na década de 70 , suas dimensões eram tão grandes que foram batizadas com o nome de "o tijolo" (the brick).

Palavras chave: Eletrônica; digital microprocessadores; circuitos; calcular.

\section{Introducción}

En nuestro entorno podemos encontrar una gran cantidad de equipos y sistemas que operan con energía eléctrica, ¿Son todos ellos productos de la ingeniería electrónica?, la respuesta es NO; únicamente son producto de la ingeniería electrónica los que emplean en sus circuitos dispositivos fabricados con materiales semiconductores. Los diodos, transistores y circuitos integrados son 
Tecnología y desarrollo: Electrónica digital

fabricados con materiales semiconductores en consecuencia ellos forman parte de los equipos o sistemas electrónicos.

Las señales de entrada y salida de los circuitos electrónicos las podemos clasificar en dos grandes grupos: "Analógicas" y "Digitales", la diferencia entre ellas es la manera en que se manifiestan sus cambios en el tiempo. Las primeras presentan cambios continuos en el tiempo por ejemplo, la temperatura, la velocidad y la distancia, dichas magnitudes pueden tomar un número infinito de valores dentro de un rango determinado en un intervalo de tiempo. Las segundas únicamente pueden tomar valores predeterminados por ejemplo un foco puede estar "encendido" o "apagado" (uno o cero), una persona puede tener fiebre o no tenerla (uno o cero), un automóvil puede estar en movimiento o detenido (uno o cero). Una señal analógica nos puede dar más información que una señal digital, esto se puede ver como una desventaja para las últimas, para superarlo se pueden emplear grupos de señales para representar diversos valores, por ejemplo un grupo de tres señales puede tomar los valores 000 , 001, 010, 011, 100, 101, 110 y 111, ocho valores diferentes en total, entre más señales tenga el grupo se pueden representar más valores diferentes, en general con " $n$ " señales se pueden representar $2 n$ valores diferentes. (Martínez, 2013)

Como nos podemos dar cuenta los sistemas analógicos emplean menos señales que los digitales esto es una ventaja por complejidad, costo y otras más, entonces ¿Por qué emplear sistemas digitales? La principal ventaja de los sistemas digitales es su inmunidad al ruido, esto es, pequeños cambios en su magnitud pueden ser ignorados sin afectar al sistema. Para procesar las señales digitales se emplean las funciones lógicas "AND", "OR" y "NOT", existen otras pero cualquier aplicación se puede realizar con éstas, las funciones lógicas restantes se pueden derivar de las citadas. La base matemática del procesamiento digital se la debemos a George Boole (1815 -1864), de seguro él no se imaginó el mundo actual producto de su Álgebra Booleana. (Martínez, 2013)

Los sistemas digitales son más antiguos que la electrónica, ello se debe a que podemos tener sistemas digitales que no sean electrónicos, por ejemplo, los circuitos eléctricos para la iluminación de nuestros hogares son casi siempre digitales, incluso se tiene procesamiento digital, cuando ocupamos dos interruptores para una misma lámpara, por ejemplo, en las escaleras. Dichos circuitos se tienen desde antes de la invención del transistor. En el transcurso de la historia hemos desarrollado diferentes tecnologías de circuitos digitales, podemos citar: interruptores, relevadores, diodos, diodos con 
Tecnología y desarrollo: Electrónica digital

transistores, circuitos integrados. Cada tecnología ha tenido auge en diferentes momentos de la historia, pero las nuevas tecnologías no han eliminado totalmente las anteriores, hoy se tienen aplicaciones con las diferentes tecnologías. (Martínez, 2013)

El empleo de los circuitos integrados también ha evolucionado con el tiempo y cada vez se pueden incluir más compuertas en un circuito integrado del mismo tamaño, los primeros únicamente tenían cuatro o seis compuertas, hoy en día tienen cientos de miles. De los circuitos integrados más recientes tenemos: microprocesadores, microcontroladores y los arreglos de compuertas lógicas configurables o programables en campo (FPGAs, por sus siglas en inglés). (Martínez, 2013).

Lo más impresionante es que gracias al poder de cómputo de los circuitos electrónicos digitales que contiene un teléfono celular, éste se ha vuelto una herramienta indispensable en nuestra vida diaria para tomar fotografías, conectarnos a Internet, mantener a nuestros amigos y familiares informados acerca de lo que hacemos y dónde nos encontramos durante el día y también para mantenernos informados de lo que ellos hacen y a donde van. Lo más hilarante de todo es que la función menos utilizada en los teléfonos celulares es aquella para la cual fueron creados, que es hablar por teléfono. (Yepez, 2013)

\section{Materiales y Métodos}

Se realizó una revisión selectiva sobre el tema objeto de estudio. La consulta se efectuó dos proceso de evolución: primero, desde la perspectiva de la electrónica y componentes que lo involucra y segundo, dirigida particularmente al tema de la electrónica digital de esta manera se obtuvo una gran cantidad de documentos generales y específicos que permitieron comprender mejor al objeto de estudio particular: la electrónica digital, en el contexto de la electrónica y sus avances. En resumen la metodología empleada fue una revisión bibliográfica documental de tipo no experimental.

La Electrónica Digital es la parte de la Electrónica que trabaja con variables discretas. Este hecho implica que un pequeño cambio en alguna de las variables del circuito (siempre que no cambie su valor discreto) no producirá un cambio apreciable en el comportamiento del circuito. Es decir, el comportamiento del circuito no depende del valor exacto de la señal. (Comunidad Educativa Autónoma de Galicia, 2014) 
Tecnología y desarrollo: Electrónica digital

Un mismo circuito electrónico, dependiendo de la zona de operación que interese, tiene su operación analógica y digital. No obstante, debemos conocer exactamente el valor de la señal de entrada (su valor continuo) para poder dar un valor a la señal de salida. (Comunidad Educativa Autónoma de Galicia, 2014)

La electrónica digital trabaja con números. La información está en los números y no en la forma de la señal. Cualquier señal se puede convertir a números y recuperarse posteriormente.

Un circuito digital realiza manipulaciones sobre los números de entrada y genera unos números de salida. En consecuencia, se podría decir que parece que los circuitos digitales son inteligentes, aunque esto no es cierto, ya que no tienen capacidad de pensar por cuenta propia, sino que están programados por la persona que los diseño así. (Tranzer in electronica digital, 2009)

Esto de una manera más completa quiere decir que los dispositivos analógicos procesan las señales variantes en el tiempo que pueden adquirir cualquier valor a lo largo de un intervalo continuo de voltaje, corriente un otra medida.

Una señal digital se hace para tomar en cualquier instante solo dos valores denominados 0 y 1 (o bajo=low. $y$ alto=high).

Apenas a partir de estos últimos 20030 años la revolución digital se ha extendido a casi todos los aspectos de la vida. Para demostrarlo, los ejemplos se pueden ver en el hecho de que muchos sistemas analógicos ahora se han vuelto digitales. (Tranzer in electronica digital, 2009)

Entonces ¿por qué ha surgido una revolución digital? Pues entre las muchas razones para dar preferencia a los circuitos digitales sobre los analógicos están:

Facilidad de diseño. El diseño digital o también conocido como "diseño lógico", como su nombre lo indica es lógico. No se necesitan habilidades matemáticas especiales, y para analizar el comportamiento de los circuitos lógicos solo hace falta visualizarse mentalmente sin tener conocimientos previos sobre cómo funcionan los transistores, capacitores u otros dispositivos que requieren de cálculos matemáticos para modelarse. (Tranzer in electronica digital, 2009)

- Flexibilidad y funcionalidad. Una vez que un problema se ha reducido a su forma digital se puede resolver utilizando pasos lógicos. Por ejemplo, se puede diseñar un circuito que mezcle o 
Tecnología y desarrollo: Electrónica digital

codifique una voz grabada que sea absolutamente indescifrable para cualquiera que no tenga su "clave". Este principio se utilizó para codificar mensajes, pero luego existieron personas que notaron efectos interesantes en los sonidos generados y dio el paso para la creación de un dispositivo llamado vocear (más sencillo es lo que utilizan los Data Punk para hacer su música). (Tranzer in electronica digital, 2009)

- Economía. Los circuitos digitales proporcionan mucha funcionalidad en un reducido espacio. ¡Estos pueden integrarse en un solo chip y fabricarse masivamente a un bajo costo, inclusive haciendo posible la creación de productos desechables como calculadoras de bolsillo, relojes digitales de pulsera y tarjetas musicales de felicitación! (Tranzer in electronica digital, 2009)

- Avance tecnológico constante. Cuando se diseña un sistema digital, se sabe que habrá una tecnología más rápida, económica o superior en poco tiempo.

Por otra parte, dentro de los circuitos digitales, existe una división en dos grandes grupos: circuitos combinacionales y circuitos secuenciales. Los circuitos combinacionales se caracterizan por el hecho de que las salidas únicamente dependen de la combinación de entradas y no de la historia anterior del circuito; por lo tanto, no tienen memoria y el orden de la secuencia de entradas no es significativo. Los circuitos secuenciales se caracterizan por el hecho de que las salidas dependen de la historia anterior del circuito además de la combinación de entradas; por lo que estos circuitos sí disponen de memoria y el orden de la secuencia de entradas sí es significativo. (Comunidad Educativa Autónoma de Galicia, 2014)

\section{Ventajas y desventajas de los sistemas digitales}

El mejor argumento a favor de la mayor flexibilidad de los sistemas digitales se encuentra en los actuales ordenadores o computadoras digitales, basados íntegramente en diseños y circuitos digitales. Las principales ventajas de los sistemas digitales respecto a los analógicos son:

1. Mayor facilidad de diseño, púes las técnicas están bien establecidas.

2. El ruido (fluctuaciones de tensión no deseadas) afecta menos a los datos digitales que a los analógicos), ya que en sistemas digitales sólo hay que distinguir entre valor alto y valor bajo. 
Tecnología y desarrollo: Electrónica digital

3. Las operaciones digitales son mucho más precisas y la transmisión de señales es más fiable porque utilizan un conjunto discreto de valores, fácil de diferenciar entre sí, lo que reduce la probabilidad de cometer errores de interpretación.

4. Almacenamiento de la información menos costoso

Los sistemas digitales presentan el inconveniente de que para transmitir una señal analógica debemos hacer un muestreo de la señal, codificarla y posteriormente transmitirla en formato digital y repetir el proceso inverso. Para conseguir obtener la señal analógica original todos estos pasos deben hacerse muy rápidamente (aunque los sistemas electrónicos digitales actuales trabajan a velocidades lo suficientemente altas como para realizarlo y obtener resultados satisfactorios). (Landin, 2014)

\section{Álgebra De Boole}

En 1854, en su obra An Investigation of the Laws of Thought el matemático inglés George Boole desarrolló un álgebra que afecta a conjuntos de dos tipos: conjunto vacío y conjunto lleno. Este álgebra se puede extrapolar a sistemas que tienen dos estados estables, " 0 " y "1", encendido y apagado, abierto y cerrado, ... Boole nunca conoció las tremendas repercusiones de su álgebra, pues no fue hasta 1939, en que Claude. E. Shannon publicó su obra A Symbolic Analysis of Relay and Switching Circuits, cuando se estableció la relación existente entre el álgebra de Boole y el estudio de los circuitos electrónicos. (Landin, 2014)

\section{Sistemas de numeración}

\section{- Sistema Decimal}

Su origen lo encontramos en la India y fue introducido en España por los árabes. Es un sistema de base 10; i.e. emplea 10 caracteres o dígitos diferentes para indicar una determinada cantidad: $0,1,2$, $3,4,5,6,7,8$, y 9 . Es un sistema posicional, de manera que el e valor de cada cifra depende de su posición dentro de la cantidad que representa. $2165=20103+1 \mathrm{o} 102+60101+5 \mathrm{o} 100=2000+100+60$ +5 (Landin, 2014) 


\section{- Sistema Binario}

Los ordenadores y en general todos los sistemas que utilizan electrónica digital utilizan el sistema binario. En la electrónica digital sólo existen dos estados posibles $\left(\begin{array}{lll}1 & \text { o } & 0\end{array}\right)$ por lo que interesa utilizar un sistema de numeración en base 2, el sistema binario. Dicho sistema emplea únicamente dos caracteres, 0 y 1 . Estos valores reciben el nombre de bits (dígitos binarios). Así, podemos decir que la cantidad 10011 está formada por 5 bits.

Al igual que en el sistema decimal, la información transportada en un mensaje binario depende de la posición de las cifras. Por ejemplo, en la notación decimal, sabemos que hay una gran diferencia entre los números 126 y 621. ¿Cómo sabemos esto? Porque los dígitos (es decir, el 6, el 2 y el 1) se encuentran en posiciones diferentes. (Landin, 2014)

Los grupos de bits (combinaciones de ceros y unos) se llaman códigos y se emplean para representar números, letras, instrucciones, símbolos. Cada bit dentro de una secuencia ocupa un intervalo de tiempo definido llamado periodo del bit. En los sistemas digitales todas las señales han de estar sincronizadas con una señal básica periódica llamada reloj. (Landin, 2014)

\section{- Puertas Lógicas}

Las operaciones matemáticas habituales, en el mundo de las matemáticas binarias, son operaciones "complicadas". Existen operaciones más sencillas llamadas operaciones lógicas. Las operaciones lógicas pueden hacerlas algunos circuitos construidos con transistores. Este tipo de circuitos se llaman puertas lógicas. (Landin, 2014)

Por consiguiente, una puerta lógica no es ni más ni menos que un circuito electrónico especializado en realizar operaciones booleanas. Las puertas lógicas fundamentales son tres AND, OR y NOR): Combinando algunas de las puertas anteriores podemos obtener otras nuevas (NAND, NOR, XOR, XNOR). (Landin, 2014)

\section{Conclusiones}

Desde que la electrónica digital se basa, como su nombre lo insinúa, en las señales binarias que se envían a través de compuertas lógicas y otros circuitos integrados, se aplica en la construcción de múltiples circuitos para diferentes aparatos de uso común como computadoras, refrigeradores, 
Tecnología y desarrollo: Electrónica digital

televisiones, estéreos, celulares, dispositivos MP3, y una larga lista de etcéteras. Asimismo, los Circuitos Digitales permitieron el desarrollo tecnológico de las Computadoras, las redes de Datos y los sistemas de comunicación. Los elementos básicos son las compuertas Digitales: Compuertas AND, OR, NOT, NOR, XOR que implementan las funciones lógicas. El sistema de numeración empleado es el Binario, consta de dos dígitos: 0 y 1. (Granados, 2012)

\section{Recomendaciones}

Profundizar en el estudio de la electrónica digital y en cada uno de sus componentes que lo involucran para una aplicación específica, tomando en cuenta las consideraciones prácticas necesarias como los circuitos o la programación. (Granados, 2012)

Utilizar los estudios de las modulaciones, para ofrecer un soporte matemático bastante estudiado y por generar resultados con la mayor eficiencia.

Explotar las propiedades que ofrece esta tipología, como sus componentes, que permite implementar procesos modernos. Asimismo, la utilización de métodos modernos, que permitan compensar una solución de estabilidad y eficiencia dentro del desarrollo de la electrónica digital. (GRANADOS, 2012)

\section{Referencias Bibliográficas}

Dr. Eduardo Cabal-Yépez, Departamento de Estudios Multidisciplinarios, División de Ingenierías, Campus Irapuato-Salamanca, Universidad de Guanajuato (09 de enero del 2003). Disponible en: http://www.ugto.mx/eugreka/contribuciones/30-tu-vida-sin-electronica-digital, Recuperado: 20 de enero del 2017.

Mtro. Juan Antonio Navarro Martínez, Profesor de Tiempo Completo del Departamento de Computación, Electrónica y Mecatrónica en la UDLAP. (20 de marzo del 2013), disponible, en: http://blog.udlap.mx/blog/2015/03/queeslaelectronicadigital/, Recuperado: 17 de enero del 2017.

COMUNIDAD EDUCATIVA AUTÓNOMA DE GALICIA, (14 de julio del 2014), disponible en: http://www.edu.xunta.gal/centros/iesfelixmuriel/system/files/, Recuperado: 17 de Enero del 2017. 
Tecnología y desarrollo: Electrónica digital

Ing. Pedro Landin, (28 de junio del 2014), disponible en: http://pelandintecno.blogspot.com.es/p/blogpage.html, recuperado: 17 de enero del 2017.

Tranzer in electronica digital, (10 de septiembre del 2009), disponible en: https://digitronica.wordpress.com/2009/09/10/electronica-analogica-vs-electronica-digital/, recuperado: 18 de enero del 2017.

Profesor José Juan Hernández Granados, (20 de enero del 2012), disponible en: http://electronicinformatica.blogspot.com/2012/01/conclusion-electronica-digital.html, recuperado: 18 enero del 2017. 